\title{
Postprandial motor response of the small intestine to enteral feeds in preterm infants
}

\author{
W M BISSET, J WATT, ${ }^{*} \mathrm{R}$ P A RIVERS, ${ }^{*}$ AND P J MILLA \\ Institute of Child Health and ${ }^{*}$ Department of Paediatrics, St Mary's Hospital Medical School, London
}

SUMmARY The postprandial motor response to enteral feeds of the small intestine of preterm infants is dependent on the bolus volume of feed that the infants receive and the number of days over which enteral feeds have been given. The postconceptional age of the infant is not an important determinant. The early introduction of enteral nutrition to preterm infants is therefore likely to enhance the motor response of the small intestine to feeds.

In the very preterm infant immaturity of gastrointestinal function frequently precludes successful postnatal adaptation, so that nutritional requirements cannot all be taken by the enteral route. For the efficient assimilation of luminal nutrients the integration of adequate digestive, absorptive, and motor function is required. Absorptive ${ }^{1}$ and digestive ${ }^{2}$ function are moderately well developed by 28 weeks' gestation, but the development of adequate motor function lags many weeks behind, ${ }^{3}$ and thus limits tolerance to enteral feeds. ${ }^{4}$ Motor function requires the integrated maturation of enteric nerves, endocrine cells, and smooth muscle cells.

Though enteral feeding enhances the development of intestinal humoral controls in the human infant,${ }^{5}$ and mucosal digestive function in animals, ${ }^{6}$ little is known about the effect of feeding on the development of intestinal motor activity in preterm human infants.

Small intestinal motor activity can be divided into two distinct phases. Between meals a cyclical fasting or interdigestive pattern of motor activity occurs, which is disrupted after a meal and replaced by continuous activity that promotes mixing and segmentation of the luminal contents; the postprandial pattern (fig 1).

The fasting pattern in the child and the adult is characterised by the appearance about every 90 minutes of a highly organised band of phasic contractions that passes from the stomach along the small intestine to the ileum. The phasic contractions (phase III) are preceded by a period of irregular contractile activity (phase II) and followed by a period of quiescence (phase I); the whole recurring cycle is referred to as the migrating myoelectric or motor complex. Previous studies of fasting small intestinal motor activity in preterm infants have shown a clear gestationally dependent programme of maturation, ${ }^{7}$ which is characterised by: low amplitude random contractions without obvious organisation (28-32 weeks' postconceptional age); short (four minute) bursts of organised contractile activity or clustered phasic activity (28-35 weeks); longer (12 minute) periods of propagated motor activity or prolonged phasic activity (34-36 weeks); and migrating motor complex activity occurring every 25 minutes (37-42 weeks).

The increase in the magnitude and the degree of organisation of fasting motor activity with increasing postconceptional age is probably related to the acquisition and development of enteric neural networks.

The postprandial pattern that develops after a meal normally lasts for 3-4 hours in the adult, at which time the fasting pattern returns. The aim of the present study was to define the longitudinal development of the motor response to food of infants born prematurely.

\section{Subjects and methods}

Nine preterm infants aged $28-42$ postconceptional weeks, weight $830-3260 \mathrm{~g}$, were studied, eight longitudinally (two subjects on four occasions, two on three occasions, and four on two occasions) and one on a single occasion (a total of 23 separate occasions). All but the most severely ill infants were included in the study and no infant was studied on more than four occasions. The study was approved by St Mary's Hospital district ethics committee. 


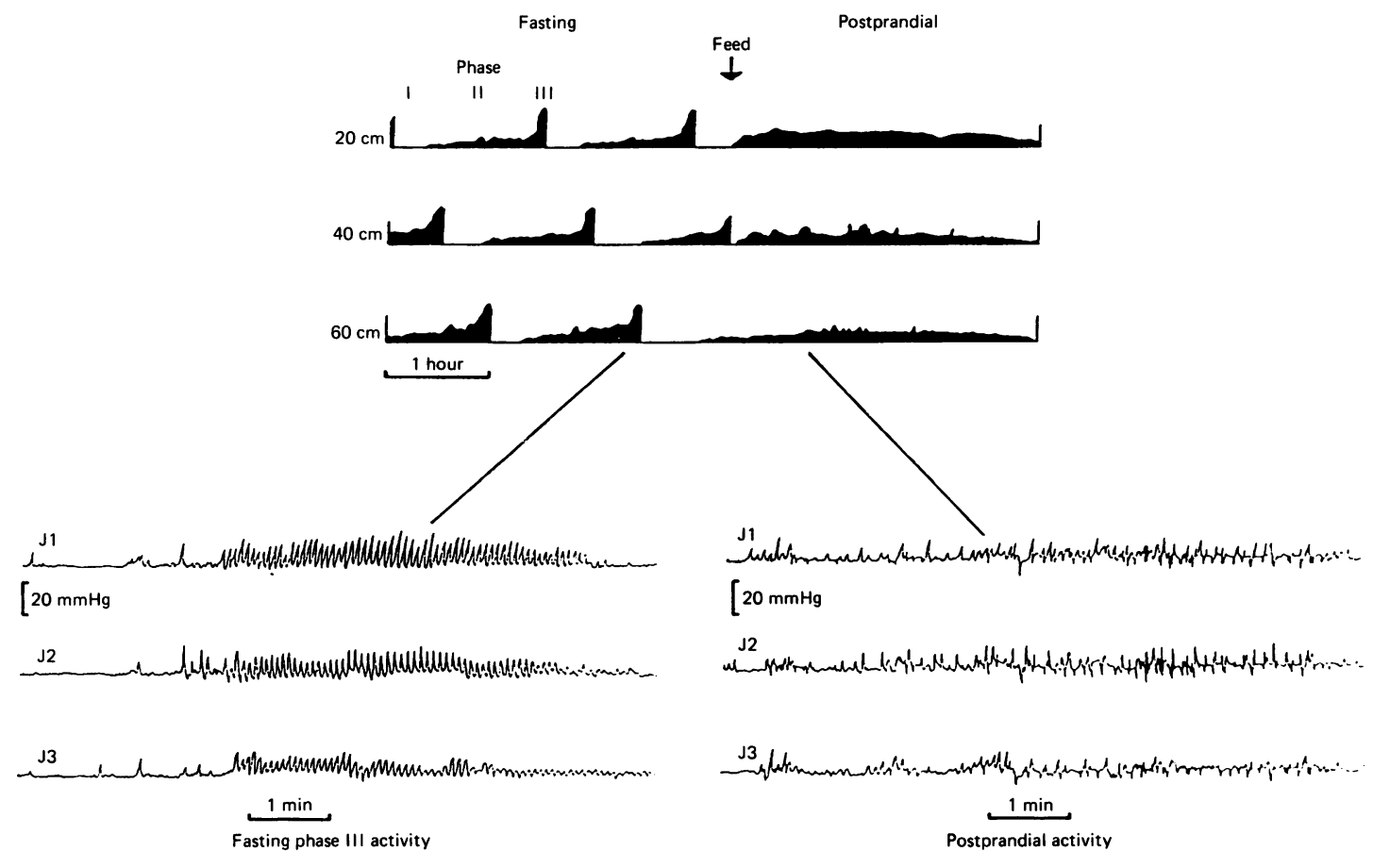

Fig 1 A compressed recording of small intestinal motor activity from a 6 year old child with an expanded view of a period of fasting and postprandial activity from three different sites in the jejunum $(\mathrm{J} 1, \mathrm{~J} 2, \mathrm{~J} 3) 5 \mathrm{~cm}$ apart.

Informed consent was obtained from the parents of each infant before the study.

The infants' ages are given as the gestational age, that is the period of gestation at birth, or as the postconceptional age, which is the gestational age plus postnatal age.

Small intestinal motor activity was measured using a perfused catheter manometric technique. Soft polyvinyl chloride multilumen catheters external diameter $1.5 \mathrm{~mm}$, internal diameter $0.7 \mathrm{~mm}$ (double channel) or $0.5 \mathrm{~mm}$ (triple channel) (Dural Plastics), were used in all studies. The distal perfusion ports were separated by $2.5 \mathrm{~cm}$. In the first 13 studies the double lumen catheter was used. With the development of an improved technique of tube manufacture we were able to use a triple lumen catheter in the last 10 studies. The manometric catheter was perfused with sodium chloride 150 $\mathrm{mmol} / \mathrm{l}$, at a rate of $0.4 \mathrm{ml} / \mathrm{channel} /$ hour delivered by a syringe pump system (Harvard Infusion Pump). Pressure changes were measured by external Luerlock Mark 3 pressure transducers (Gaeltec), and the signal was displayed on a multichannel oscillographic chart recorder (Washington MD4).

The manometric catheter was advanced through the nose into the stomach of each subject and, while pressure changes were being recorded, further advancement through the pylorus and into the duodenum was monitored by the characteristic change in the frequency of motor activity from 3 cycles/min in the stomach to $12 \mathrm{cycles} / \mathrm{min}$ in the duodenum. In four of the smaller infants the position of the tube was confirmed by radiographic examination, which was carried out for clinical reasons. In each case the tip of the catheter was at the duodenojejunal flexure.

\section{INFANT FEEDS}

As we believed that it would be unethical to alter the management of the patients for the purpose of this study, the infants received a bolus feed equal to that which they were previously receiving, or (in those on continuous feeds) a volume equivalent to one hour's feed was given as a bolus. Some infants received expressed breast milk, some formula feed, and others received a mixture of the two. The energy density of all the feeds was similar. The very preterm infants received their feed nasogastrically and the more mature infants were fed orally. The nasogastric feeds were completed within 10 minutes 
and the oral feeds within 20 minutes. The recordings of small intestinal motility were made continuously both before, during, and after each feed. The study was designed to determine the effect of feeding on gut motor activity and not to compare one feed with another or to determine differences resulting from the mode of feed delivery.

\section{ASSESSMENT OF MOTILITY}

The infants were all fasted before the study. In most infants this was for between 4-24 hours, but in a few of the smaller infants, who were being totally enterally fed, only a two hour fast was possible if hypoglycaemia was to be prevented. During each study small intestinal motor activity was recorded for at least four hours. After an initial one hour period of fasting motor activity the infant was fed, as described above, and the pattern of postprandial motor activity was noted. The postprandial activity was characterised by the development of apparently random non-propagative activity that disrupted the basal fasting pattern. The length of postprandial activity was defined as the length of time taken from the beginning of a feed until the disappearance of the postprandial pattern and the return of the fasting pattern of activity.

The frequency of duodenal contractile activity during periods of continuous motor activity was measured by inspection of the manometric recording for each of the 23 studies.

\section{STATISTICAL ANALYSIS}

The association between variables is expressed by the correlation coefficient $(r)$ and the significance of this association was derived from the $F$ score by linear regressional analysis. Multiple regressional analysis was used to assess the significance of factors influencing the length of postprandial motor activity. Means are expressed plus or minus one standard deviation. The significance of the differences between normally distributed variables was tested by the paired Student's $t$ test. Probabilities of 0.05 or less were regarded as significant.

\section{Results}

POSTPRANDIAL PATTERN

After feeding one of three postprandial responses occurred (fig 2):

(i) in the smallest infants ( $<31$ weeks' postconceptional age) who were receiving low volumes of continuous enteral feed, postprandial activity did not occur and if a fasting pattern was seen this was not disrupted by the enteral feed. ${ }^{7}$ Fig 2 (a) shows a postprandial pressure recording from such an infant
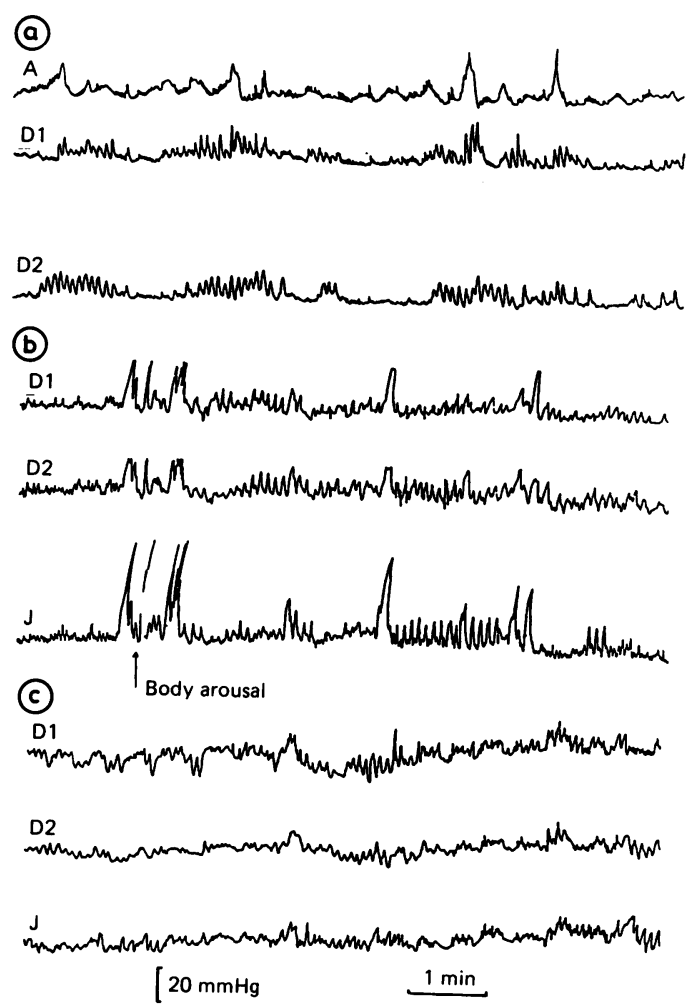

Fig 2 Small intestinal motor activity recorded at multiple sites, within 15 minutes of the start of a bolus feed in infants of (a) 30 weeks' postconceptional age (b) 35 weeks, and (c) full term. The traces are recorded from $A$ (antrum of stomach), $D$ (duodenum), and $J$ (jejunum).

with clustered phasic activity and a complete lack of any postprandial response.

(ii) In infants of 31-35 weeks' postconceptional age, larger volumes of feed were given that induced some degree of postprandial activity, but often the underlying fasting pattern remained. In fig 2(b) a period of clustered phasic motor activity is superimposed on a background of more random postprandial activity.

(iii) Finally, in the older infants ( $>35$ weeks' postconceptional age) who were receiving large volumes of bolus feed, there was complete disruption of the cyclical fasting activity with continuous postprandial activity lasting up to two hours as shown in fig 2(c). Towards the end of the postprandial period, however, fasting activity returned giving a pattern similar to that seen in the less mature infants. 
FREQUENCY OF CONTRACTILE ACTIVITY

The mean frequency of maximum motor contraction in the duodenum estimated from all 23 recordings during the postprandial phase of activity of 11.5 $(0 \cdot 6) \mathrm{cycles} / \mathrm{min}$ was not significantly different from that found in the fasting state $(11.4(0.6)$ cycles/ $\min$ ).

\section{MULTIPLE REGRESSION MODEL}

A multiple regression model was developed to study the factors that might influence the postprandial response. The length of postprandial activity was used as the dependent variable and postconceptional age, bolus feed volume, and time fed enterally, as the independent variables. Fig 3 shows

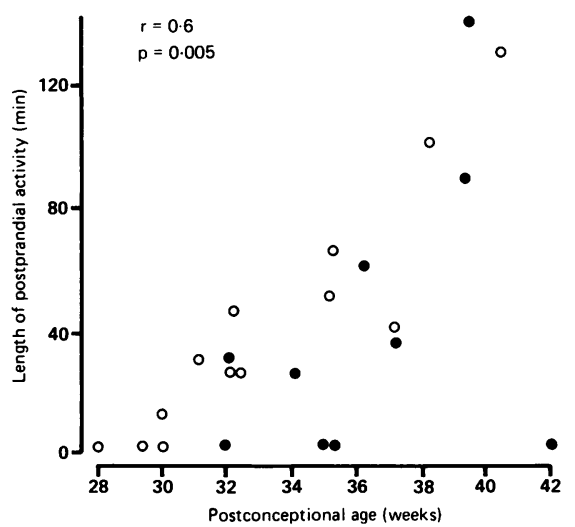

Fig 3 The length of postprandial motor activity compared with the postconceptional age in 23 infants. Open circles denote those born at $<29$ weeks' gestation, and closed circles those born at $\geqslant 29$ weeks.

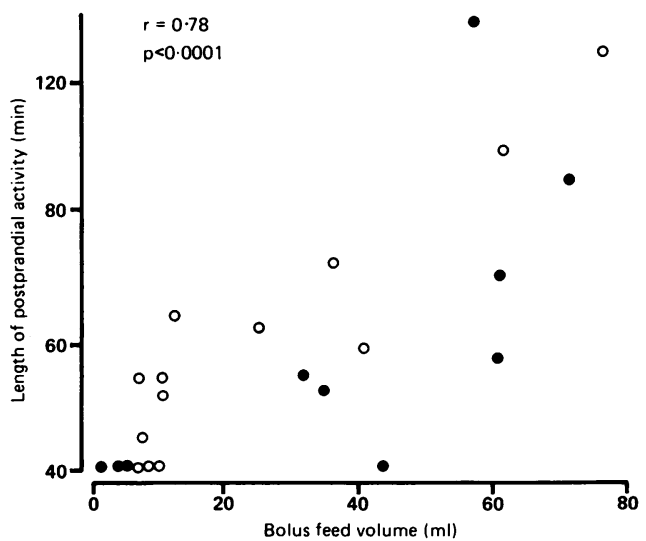

Fig 4 The length of postprandial motor activity plotted against the volume of bolus feed in 23 infants. Open circles denote those born at $<29$ weeks' gestation, and closed circles those born at $\geqslant 29$ weeks. that with increasing postconceptional age the length of postprandial activity following a feed increased $(r=0 \cdot 6)$. This increase may, however, reflect the fact that the more mature infants were receiving larger volumes of feed and using the multiple regression model described above it can be seen that postconceptional age $(p=0.37)$ contributes little, while the volume of feed $(p=0.005)$ seems to be the most important determinant of the correlation. This is reflected in fig 4 where the length of postprandial activity plotted against bolus feed volume shows an improvement in the correlation coefficient with the length of activity from 0.6 to 0.78 .

In both figs 3 and 4 those infants born before 29 weeks are differentiated from those born at 29 weeks or later. For a given postconceptional age (fig 3 ) those born more prematurely seemed to have better developed postprandial activity, but this difference is not significant $(p=0 \cdot 07)$. For a given feed volume, however (fig 4), those born more prematurely - and thus of greater postnatal agehave a significantly longer postprandial response $(p=0.03)$. When the length of time that each infant had been on enteral feeds was plotted against the duration of postprandial activity (fig 5) an even stronger correlation between these variables was seen $(r=0 \cdot 89, p<0 \cdot 0001)$. In addition, the previous differences between the two groups of preterm infants had disappeared. The length of postprandial activity also increased progressively from that observed in the continuously fed infants to that seen in those receiving four hourly bolus feeds $(r=0.66$, $p=0 \cdot 001$ ). Thus the multiple regressional model clearly shows that the period of time during which

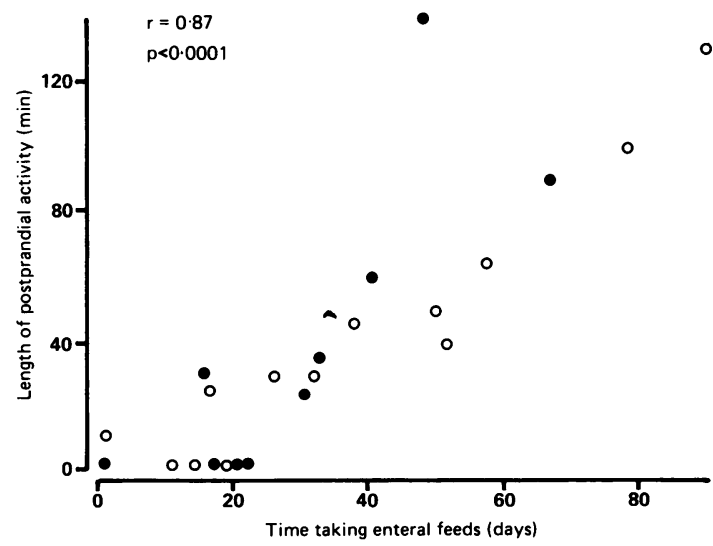

Fig 5 Plot of the length of postprandial motor activity against the length of time on enteral feed in 23 infants. Open circles denote those born at $<29$ weeks' gestation, and closed circles those born at $\geqslant 29$ weeks. 
Table Correlation of variables with length of postprandial activity

\begin{tabular}{ll}
\hline Variable & Correlation \\
\hline Postconceptional age & 0.60 \\
Increasing feed interval & 0.66 \\
Postnatal age & 0.78 \\
Volume of bolus feed & 0.78 \\
Volume of daily feed & 0.80 \\
Time receiving enteral feeds & 0.87 \\
\hline
\end{tabular}

p Value $<0.005$ in all cases.

the infant has been taking enteral feeds and-to a lesser extent-the actual bolus feed volume, are important determinants of the postprandial motor response, while the postconceptional age of the infant seems to be of lesser importance. The factors that correlate with postprandial activity are summarised in the table.

\section{Discussion}

Unlike fasting motor activity, which appears to be tightly controlled by programmed actions of the enteric nervous system, postprandial activity represents the motor response of the gut to food and will vary according to the nature of the stimulus (the feed) and the ability of the gut to respond to that stimulus. Thus as the volume of enteral feed increases with increasing postconceptional age, so the degree of postprandial activity might also increase. Our data show that this is indeed the case, and from the analysis it would seem that the increased length of time taking enteral feeds and the increased feed volume are the principal determinants of this change, and postconceptional age is not important.

Disruption of fasting small intestinal motor activity and the induction of a postprandial pattern has been associated with the release of intestinal polypeptide hormones such as gastrin, cholecystokinin, ${ }^{8}$ and peptide YY. ${ }^{9}$ The magnitude and length of the postprandial motor response is associated with the volume and energy density of the feed delivered. ${ }^{10} 11$

If a certain degree of response is required to disrupt the fasting pattern of activity and initiate postprandial activity, this would account for the findings in the present study in infants receiving low volumes of feed in whom no postprandial response was seen. In those receiving larger volumes an intermediate response occurred with superimposed fasting and postprandial activity, and in those receiving large volumes of enteral feed there was complete disruption of the fasting pattern and replacement by postprandial activity for over an hour. The appearance of the intermediate response towards the end of a prolonged period of postprandial activity could result from the humoral response falling as the feed was digested and absorbed.

Our data show that the length of time that an infant has been taking enteral nutrition seems to be the most important factor in determining the length of postprandial activity. This suggests that the hormonal response that is responsible for the initiation of postprandial activity is enhanced by increased exposure of the gut to enteral nutrition. Support for this hypothesis is provided by previous studies in humans, ${ }^{512}$ which show that the hormonal response to an enteral feed increased as the time taking enteral feeds increased. We did not record the cumulative volume of feed delivered to each of the infants as previous studies have shown that only low cumulative volumes $(<96 \mathrm{ml})$ of feed are needed to produce a pronounced rise in the mean plasma concentrations of many polypeptide gut hormones, including some associated with postprandial activity, above the newborn concentrations. ${ }^{13}$ The use of a factor combining length of time taking feed and feed volume, which might be most closely related to cumulative feed volume, did not increase the correlation with the length of postprandial activity above that seen using only length of time on feed. Though our data might suggest that there is an enhanced humoral response to enteral feeds related to the length of time that an infant has been fed, there are remarkably few data from studies which explore this possibility. Longitudinal studies are required to confirm that this is indeed the case.

The optimal feeding regimens for preterm infants continue to be debated. Our data suggest that as enteral feeding enhances the postprandial humoral response it should be started as early as possible. It is quite clear that total parenteral nutrition delays these beneficial effects. ${ }^{5}$ It is possible that even small nutritionally insignificant volumes of feed could be of benefit to infants who could not tolerate full volume feeds. ${ }^{13}$ The feeds may not only be beneficial in promoting villus absorptive ${ }^{1}$ and digestive function, ${ }^{6}$ but also in enhancing the humoral responses of the gut necessary for postprandial motor activity. ${ }^{513}$ It might also be predicted that the type of feed and the mode of delivery of the feed will have some influence on the postprandial response. It is likely, however, that because of interindividual variations in this resonse, large numbers of infants would have to be studied to show significant differences between feeding regimens.

Thus while the development. of fasting motor activity in preterm infants is gestationally dependent 
and is related to the maturation of enteric and central neural connections, ${ }^{7}$ the postprandial motor response in contrast seems to be mainly influenced by how much and for how long the infant has been fed. It would, therefore, seem that exposure of the gut to feeds enhances the humorally mediated postprandial response.

W M B and P J M thank Duphar B V, Netherlands, for their continued interest and financial support, and Smith, Kline, and French for their help with the purchase of equipment.

\section{References}

${ }^{1}$ McNeish AS, Ducker DA, Warren IF, Davies DP, Harran MJ, Hughes CA. The influence of gestational age and size on the absorption of D-xylose and D-glucose from the small intestine of the human neonate. In: Elliott K, Whelan J, eds. Development of mammalian absorptive processes. Ciba Foundation Symposium 70. Amsterdam: Excerpta Medica, 1979:267-80.

2 Grand RJ, Watkins JB, Torti FM. Development of the human gastrointestinal tract. Gastroenterology 1976;70:790-810.

3 McLain CR. Amniography studies of the gastrointestinal motility of the human fetus. Am J Obstet Gynecol 1963;86: 1079-87.

4 Dunn PM. Intestinal obstruction in the newborn with special reference to transient ileus associated with respiratory distress syndrome. Arch Dis Child 1963;38:459-67.

5 Lucas A, Adrian TE, Christofides N, Bloom SR, Aynsley-
Green A. Plasma motilin, gastrin, and enteroglucagon and feeding in the human newborn. Arch Dis Child 1980;55:673-7.

${ }^{6}$ Hughes CA, Dowling RH. Speed of onset of adaptive mucosal hypoplasia and hypofunction in the intestine of parentally fed rats. Clin Sci 1980;59:317-27.

${ }^{7}$ Bisset WM, Watt J, Rivers RPA, Milla PJ. The ontogeny of fasting small intestinal motor activity in the human infant. Gut 1988;29:483-8.

8 Wingate DL, Pearce EA, Hutton M, Dand A, Thomson HH, Wunsch E. Quantitative comparison of the effects of cholecystokinin, secretin and pentagastrin on gastrointestinal myoelectric activity in the conscious fasted dog. Gut 1978;19: 593-601.

9 Adrian TE, Smith HA, Calvert SA, Aynsley-Green A, Bloom SR. Elevated plasma peptide $Y Y$ in human neonates and infants. Pediatr Res 1986;20:1225-7.

10 Weisbrodt NW. Motility of the small intestine. In: Johnston LR, ed. Physiology of the gastrointestinal tract. Vol 1. New York: Raven Press, 1981:411-43.

11 Amarnath RP, Berseth CL, Abell TL, Perrault J, Malagelada JR. The effect of nutrient concentration on small intestinal motility in preterm infants. Gastroenterology 1986;91:1043.

12 Lucas A, Bloom SR, Aynsley-Green A. Development of gut hormone responses to feeding in neonates. Arch Dis Child 1980; 55:678-82.

13 Lucas A, Bloom SR, Aynsley-Green A. Gut hormones and 'Minimal enteral feeding'. Acta Paediatr Scand 1986;75:719-23.

Correspondence to Dr WM Bisset, Department of Child Health, Institute of Child Health, 30 Guilford Street, London WC1N 1EH.

Accepted 28 March 1989 